\title{
Assist-as-needed Impedance Control Strategy for a Wearable Ankle Robotic Orthosis
}

\author{
João Lopes, Cristiana Pinheiro \\ University of Minho \\ Guimarães, Portugal \\ a74982@alunos.uminho.pt
}

\author{
Joana Figueiredo \\ University of Minho \\ Guimarães, Portugal \\ id6003@alunos.uminho.pt
}

\author{
Luís P. Reis \\ University of Porto \\ Porto, Portugal \\ lpreis@fe.up.pt
}

\author{
Cristina P. Santos \\ University of Minho \\ Guimarães, Portugal \\ cristina@dei.uminho.pt
}

\begin{abstract}
The use of robots in rehabilitation attempts an effective, compliant, and time-efficient gait recovery while adapting the assistance to the user's needs. Assist-as-needed strategies (AAN), such as adaptive impedance control, have been reported as prominent strategies to enable this recovery effects. This study proposes an interaction-based assist-as-needed impedance control strategy for an ankle robotic orthosis that adapts the robotic assistance by changing the Human-Robot interaction stiffness. The adaptability of the interaction stiffness allows the real-time passage from passive assistance to an active one, approaching AAN gait training. The interaction stiffness was successfully estimated by linear regression of the Human-Robot interaction torque $v s$ angle trajectory curve. From the validation with seven able-bodied subjects, we verified the suitability of this adaptive impedance control for a more compliant, natural, and comfortable motion than the trajectory tracking control. Moreover, the proposed strategy considers the users' motion intention and encourages them to interact closely with the robotic device while guiding their ankle trajectory according to desired trajectories. These achievements contribute to AAN gait training.
\end{abstract}

Keywords - Human-Robot Interaction, Impedance Control, Locomotion and Actuation Systems, Robotic Rehabilitation

\section{INTRODUCTION}

All over the decade, powered and unpowered robotic orthoses have been developed to rehabilitate persons with motor injuries caused by cardiovascular and neurological disorders [1]. Stroke is the World's second leading cause of death and third leading cause of motor disability [2]. The ankle is the most affected articulation in stroke survivors, who commonly present drop foot and an energetically cost hemiparetic gait [3], [4]. The use of robotic assistive devices on gait rehabilitation pretends to act as an aid in these patients' motor recovery, allowing a therapy sustained in repetitive and personalized gait training.

Depending on the level of disability, repetitive gait training is crucial for firsts therapy sessions [1]. However, as the patient progresses in the rehabilitation, this training becomes unappropriated to the users' needs, imposing a gait pattern and not promoting active cooperation from the patient nor personalized assistance [5]. The need arose for assist-as-needed (AAN) control strategies, in which the robotic orthosis only intervenes when it is needed aiming personalized gait training [1].

This work has been supported by the FEDER Funds through the Programa Operacional Regional do Norte and national funds from Fundação para a Ciência e Tecnologia with the project SmartOs under Grant NORTE-01-0145FEDER-030386, and through the COMPETE 2020-Programa Operacional Competitividade e Internacionalização (POCI) - with the Reference Project under Grant POCI-01-0145-FEDER-006941.
AAN strategies may endow impedance controllers, capable of modulating the movement's compliance and providing patient-robot cooperation, appealing to the patient's active interaction towards effective rehabilitation. For instance, the LokoMat exoskeleton allows the adjustment of the movement's compliance by multiplying the error in angular position by a virtual coefficient $K$ (linear elastic coefficient) and the angular velocity by a virtual coefficient $B$ (linear viscous coefficient) [5]. A reference torque is created and compared with the torque produced by the patient's muscles [5]. Another relevant work is LOPES, a robot that provides the movement's compliance adjustment with two modes of assistance: the "patient-incharge", characterized by a flexible movement, and the "robotin-charge", a robotic-imposed movement [6]. The same principals of LokoMat's and LOPES's were applied to a pneumatic orthosis proposed by Hussain et al. [7], where the assistance can be modified according to the patient's impairment level. Another work was performed by Rajasekaran et al. [8] in which the orthosis impedance was modulated according to a confidence factor, that can be adjusted by the physiotherapist, and the position errors. The variation of stiffness results in different forces acting at the joint, allowing a resistance or an assistance effect [8].

However, it is still needed to investigate the impact of adjusting the stiffness of the human-robot interaction (interaction stiffness), instead of the joint's stiffness, to achieve more compliant assistance and make the robot more cooperative with the user's motion intention and participation. To tackle this challenge, this works aims to present an assist-as-needed and adaptive impedance control strategy for a wearable ankle robotic orthosis. This control strategy modulates the interaction stiffness according to the measured human-robot interaction torque; thus, following the patients' motion intention while encouraging them to actively cooperate in the therapy session, enhancing their functional rehabilitation. The controller uses the interaction torque measured through strain gauges as an effort and motion intention indicator. This control strategy allows the passage from passive assistance, in which the orthosis do not impose a therapy (active walking [1]), to active assistance, where the robotic orthosis is in charge (passive walking [1]). Moreover, this work presents an automatic study of the human-robot interaction, allowing to infer in which gait phases it is needed a stiffer or a compliant movement towards an assist-as-needed robotic rehabilitation. The human-orthosis interaction stiffness was virtually estimated with a linear fit of the human-orthosis interaction torque $v s$ angle curve, following the least square method, without introducing mechanical apparatus, reducing mechanical issues in the orthosis. Moreover, towards an adaptive impedance control, the interaction stiffness can be 
modified in real-time using a mobile application. The findings with seven able-bodied subjects highlight the suitability of this control for robotics-based gait rehabilitation since it provides a more natural and comfortable walking to the end-user, appealing to the active cooperation in the therapy, enhancing the chances of a time-effective recovery.

This paper is organized as follows: Section II presents the methodology; Section III describes the experimental procedure for the strategy validation; Section IV reports the achieved results, the respective discussion, and future perspectives; and Section $\mathrm{V}$ presents the main conclusions.

\section{ADAPTIVE IMPEDANCE CONTROL}

This work presents and validates an adaptive impedance control strategy with a wearable robotic ankle orthosis intended for post-stroke survivors. In this section, the ankle robotic orthosis is presented, as well as the control strategy description and the methodology followed for estimating the humanorthosis interaction stiffness.

\section{A. Ankle Robotic Orthosis}

The ankle robotic orthosis, presented in Fig. 1, is an electrical-based system from the H2-Exoskeleton (Technaid S.L., Spain) that provides 1 degree of freedom (DOF) in the sagittal plane for gait speeds ranging from 0.14 to $0.44 \mathrm{~m} / \mathrm{s}$. The actuation system is a flat brushless DC motor coupled to a gearbox (Fig. 1C), that provides an average torque of $35 \mathrm{Nm}$. The actuator is aligned with the human's ankle articulation, promoting effective assistance.
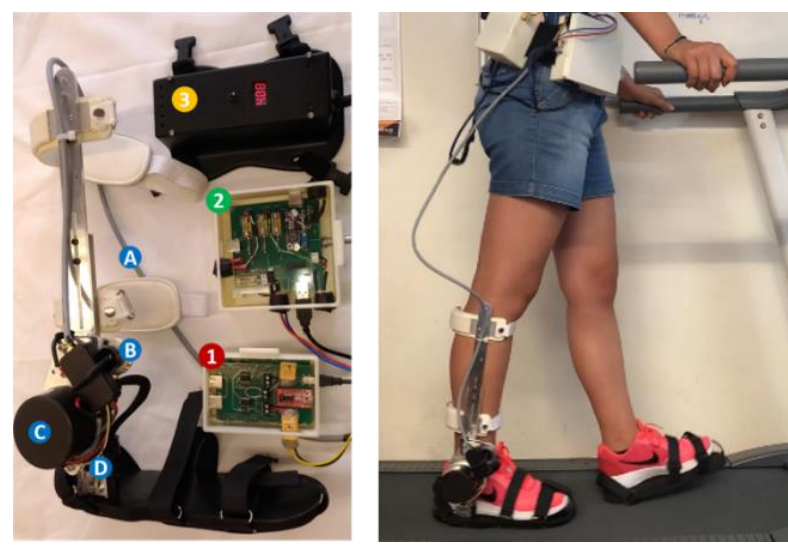

Figure 1: Ankle robotic orthosis: (A) CAN cable, (B) embedded potentiometer, (C) motor \& gearbox, (D) embedded strain gauge; (1) STM32F4-Discovery; (2) electronic board of battery; (3) LiFePO4 battery.

The orthosis contains an embedded strain gauge (Fig. 1D) and a potentiometer (Fig. 1B) that allows the continuous realtime monitorization of the human-orthosis interaction torque and angle position, respectively. The strain gauge is configurated in a Wheatstone bridge and located near to the orthosis' insole, to accurately measure the forces produced by its end-users. The potentiometer is located closer to the ankle articulation, measuring the sagittal plane angle. The robotic device is powered by a LiFePO4 battery (Fig. $1-3$ ) of $24 \mathrm{~V}$ and hierarchically controlled, dividing the control strategy into a high-, mid- and low-levels, following the proposal of Tucker et al [9]. The high-level, responsible for the central control and trajectory generator, runs in a Raspberry Pi 3 (Raspberry Pi Foundation, UK) at a $100 \mathrm{~Hz}$. The mid-level and low-level controls, responsible for the controller's implementation and CAN communication with the robotic orthosis, run in a microcontroller STM32F4-Discovery (STMicroelectronics, Switzerland) at $100 \mathrm{~Hz}$ and $1000 \mathrm{~Hz}$, respectively.

\section{B. Impedance Control Strategy}

Recent works ([7], [8]) have demonstrated the suitability of AAN strategies as a way to produce personalized assistance. The adaptive impedance control strategy is an AAN strategy intended to promote a therapy sustained in active participation and effort by the user, allowing a stiffness modulation in realtime. With that, the robotic orthosis can pass from a passive mode of assistance, in which the user interacts freely with the device, providing a self-placed walk, to an active mode of assistance, where the user follows a previously defined gait pattern. Fig. 2 presents the block diagram of the adaptive impedance control strategy implemented and validated in this work.

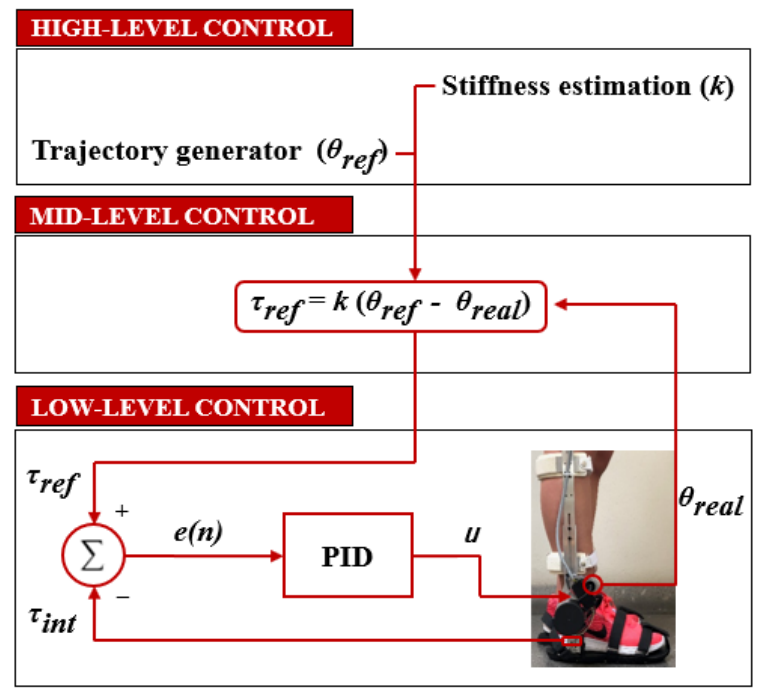

Figure 2: Block diagram of the adaptive impedance control strategy. $K$ is the interaction stiffness, adjusted in real-time through a mobile application, $\theta_{\text {ref }}$ is the reference trajectory, $\theta_{\text {real }}$ is the human/robot angle, $\tau_{\text {ref }}$ is the reference torque, $\tau_{i n t}$ the interaction torque, $e(n)$ the error and $u$ the PID command.

In the high-level, a gait reference trajectory, $\theta_{\text {ref }}$, is generated following the gait pattern described by Winter [10] for healthy subjects (see Fig. 3). The reference trajectory is sent to the midlevel control, where an impedance control law [11] is implemented. Towards an AAN strategy, the impedance control law calculates the error produced between $\theta_{\text {ref }}$ and the human/robot angle, $\theta_{\text {real }}$, in the mid-level control. In this level, a reference assistance torque, $\tau_{r e f}$, is calculated multiplying the angular error by the interaction stiffness $(k)$. This reference torque can assume two possible outcomes: zero, or near zero, if the user is performing the healthy reference trajectory with a very low error, meaning that there is no need of assistance and, thus, the orthosis acts passively, giving more freedom to the gait; and different from zero, if the user is not fully matching the healthy trajectory, meaning that the orthosis must intervene and correct the subject's gait. In this way, the orthosis acts actively, assisting the user as needed. 
The reference torque is sent to the low-level control in which a PID controller was implemented. This torque is, then, compared to the human-orthosis interaction torque, $\tau_{\text {int }}$. In this work, we acknowledge the interaction torque as the users' effort indicator, translating their active participation into a measurable variable. The force applied in the strain gauge produces a deformation according to the users' motion intention, which is converted to torque, multiplying by the moment arm.

The PID controller is responsible for generating an adequate response regarding the difference between the interaction torque and the reference torque. The controller's parameters were tuned according to the Ziegler-Nichols method [12]. For this work, the proportional coefficient was considered $K_{p}=120$, the integrative time $\left(T_{i}\right)$ as infinite and the derivative time $\left(T_{d}\right)$ as 0 .

\section{Human-Orthosis Interaction Stiffness Estimation}

The reference torque considers the interaction stiffness between the user and the orthotic device. The stiffness of a joint is the derivative of torque with respect to the angle. Dollar et al. [13] presented an approach for estimating the ankle joint stiffness regarding different gait phases instead of calculating the derivative sample by sample. The authors calculated an approximate stiffness, considered the quasi-stiffness, with a linear regression model that fits the torque $v s$ angle curve during the stance phase. In this work, a similar approach was followed but considering the user-induced interaction torque. Thus, the interaction stiffness was estimated by determining the best linear model that fits the human-orthosis interaction torque $v s$ angle trajectory curve, following the least-square method [14].

According to the least-square method, the best approximation to a certain curve is the one that produces the minimal deviations, sample by sample, to a set of data [14]. In this work, the interaction stiffness was considered the slope of the best linear fit to the human-orthosis interaction torque $v s$ angle curve, $k_{\text {int }}$. The approximation to the interaction stiffness was calculated according to (1), where $y_{i}$ is the interaction torque and $x_{i}$ is the angle trajectory.

$$
k_{\text {int }}=\frac{n \sum x_{\mathrm{i}} y_{\mathrm{i}}-\sum x_{\mathrm{i}} \sum y_{\mathrm{i}}}{n \sum x_{\mathrm{i}}-\left(\sum x_{\mathrm{i}}\right)^{2}}
$$

By analysing the human-orthosis interaction torque vs angle trajectory curve (Fig. 5), we verified the need of modulating the interaction stiffness for six phases of the gait cycle, namely: from heel-strike (HS) to foot flat (FF) - Fig. 3A, from FF to MSt (Mid-Stance) - Fig. 3B, from MSt to heel-off (HO) - Fig. 3C, from $\mathrm{HO}$ to toe-off (TO) - Fig. 3D, from TO to mid-swing (MSw) - Fig. 3E, and from MSw to a new HS - Fig. 3F. These gait phases were segmented through an adaptive finite state machine (FSM) using the angular velocity recorded with an IMU placed on the foot, as proposed in [15]. Subsequently, the interaction stiffness was averaged for each phase of the gait cycle, considering all subjects, and introduced into the mid-level control (Fig. 2).

By changing the interaction stiffness, the reference torque $\left(\tau_{\text {ref }}\right)$ can, now, assume two possible outcomes: zero, or near zero, if the interaction stiffness is set to minimum, encouraging the users to perform active participation, enhancing their recovery by applying more effort and activating their muscles; zero, or near zero, if the interaction stiffness is set to maximum but the user is able of following the healthy trajectory producing a near-zero error, meaning that no assistance is required; and different from zero, if the interaction stiffness is set to maximum and the user is not able of following the healthy trajectory, meaning that assistance is required.

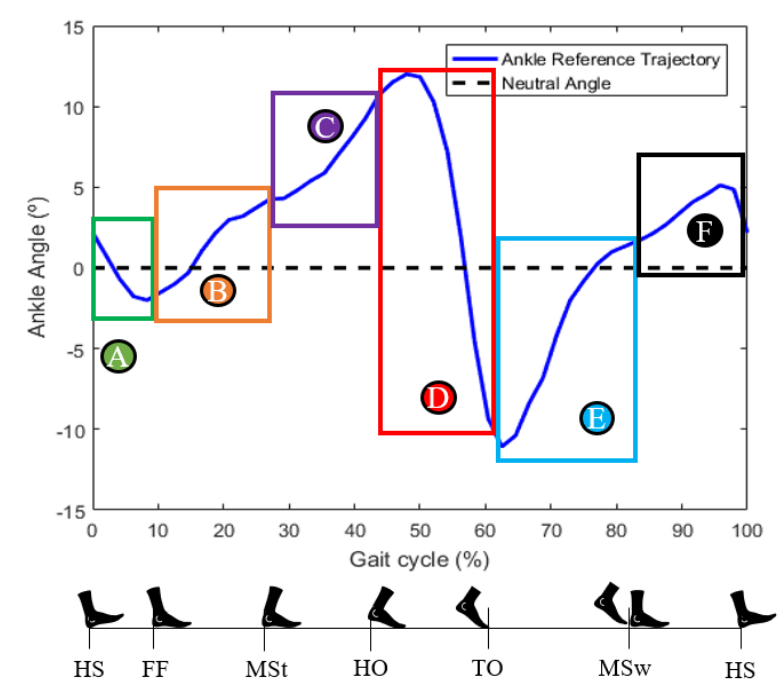

Figure 3: Healthy reference trajectory of the robotic orthosis, following the principles of Winter [11].

The interaction stiffness was estimated in offline for the six gait phases. Nonetheless, the physiotherapist may optimize this estimated parameter in real-time during the therapy session, using a user-friendly mobile application. With this real-time approach, the therapy can be user-centred and adapted to the users' needs, augmenting the chances of recovery.

\section{EXPERIMENTAL PROCEDURE}

To estimate the interaction stiffness and to validate the proposed adaptive impedance control, an experimental study was performed with able-bodied subjects walking with the ankle robotic orthosis. All subjects signed a consent form to be part of the study. Subject's rights were preserved and, as such, personal information provided was remained confidential. Data were collected at the University of Minho, Portugal.

\section{A. Participants}

Seven healthy subjects (two females and five males; body mass: $70.4 \pm 11.9 \mathrm{~kg}$; height: $170 \pm 10.1 \mathrm{~cm}$; age: $24.4 \pm 1.40$ years) without evidence of motor disorders, were recruited and accepted to participate, voluntarily, in the strategy validation.

\section{B. Experimental Protocol}

The experimental protocol was divided into two different sessions. The first session aims the kinematic and kinetic data collection for estimating the interaction stiffness and understanding in which gait phases the subjects interact more with the ankle robotic orthosis and, thus, need more assistance. For this purpose, the participants performed 3 level-ground walking trials of 1 minute using the orthotic device in an unpowered mode for three gait speeds, $0.28 \mathrm{~m} / \mathrm{s}, 0.36 \mathrm{~m} / \mathrm{s}$ and $0.44 \mathrm{~m} / \mathrm{s}$. The collected data includes foot angular velocity (from wearable IMUs), the ankle trajectory (from the embedded potentiometer) and the interaction torque (from the embedded 
strain gauge), acquired at $100 \mathrm{~Hz}$. Fig. 4 presents the experimental setup.

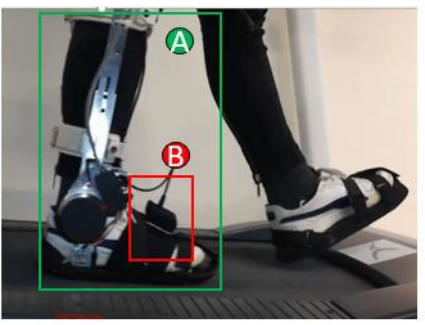

(A) Robotic Orthosis

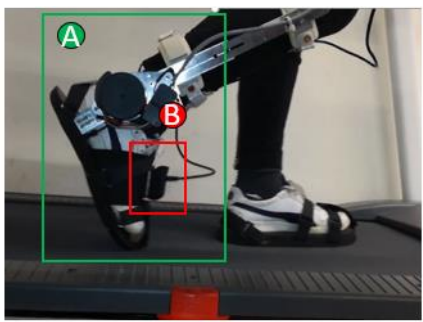

B IMU

Figure 4: Experimental setup for interaction stiffness estimation.

In the second session, the validation of the assist-as-needed impedance control strategy was performed. The subjects were instructed to walk for two continuous trials of 4 minutes. In the first minute of trial, the subjects walked with the orthosis closely to a trajectory tracking control mode, i.e., $k$ set to maximum. In the following two minutes, the interaction stiffness was modified according to the estimated values by the linear model. During these three minutes, the subjects were blind to the protocol to not influence their perception about the effects of changing the interaction stiffness. At the last minute, the subjects were instructed to stand the foot on the air and minimize their interaction with the robotic orthosis. The validation experiments were performed for both 0.28 and $0.44 \mathrm{~m} / \mathrm{s}$. The collected data include the human/orthosis real trajectory, the reference trajectory, the reference interaction torque and the human-orthosis interaction torque. During the second session, the subjects were instructed to give their perception regarding the control strategy in terms of freedom of movement, gait fluidity, and physical effort demand.

\section{Data Processing and Analysis}

For both interaction stiffness estimation and adaptive impedance control validation, kinematic (ankle angle and foot angular velocity) and kinetic data (human-orthosis interaction torque) were filtered with a zero-lag low-pass Butterworth filter with a cut-off frequency of $5 \mathrm{~Hz}$ [10].

The interaction stiffness was normalized considering the user's body mass and scaled between 0 and 1 , in which the maximum value of interaction stiffness found for each trial was considered $1 \mathrm{Nm} /{ }^{\circ} \mathrm{kg}$. Data was time-divided into gait cycles considering the angular velocity [15].

\section{RESULTS AND DISCUSSION}

\section{A. Human-Orthosis Interaction Stiffness}

In order to assess where the subjects need more assistance, the interaction stiffness was estimated following the least-square method for six phases of the gait cycle, considering the data collected in the first session. Acknowledging the interaction stiffness per gait phases allows us to infer in which part of the gait cycle the users interact more with the orthotic device.

Fig. 5 displays the human-orthosis interaction torque vs angle trajectory and the interaction stiffness estimated by the linear model for $0.28 \mathrm{~m} / \mathrm{s}$. We considered the interaction stiffness as the slope of the linear curves presented in Fig. 5, for each of the gait phases. The values were normalized by body mass and scaled between $0-1$ to allow a general analysis among subjects. Table I details the mean normalized interaction stiffness values, and the corresponding standard deviation, for the different gait moments obtained with the FSM ([15]). Moreover, the normalized interaction stiffness is also presented regarding the stance (from HS to TO) and swing (from TO to a new HS) phases, and for the double support (from HS to FF and $\mathrm{HO}$ to TO) and single support (from FF to $\mathrm{HO}$ and $\mathrm{TO}$ to HS) phases.
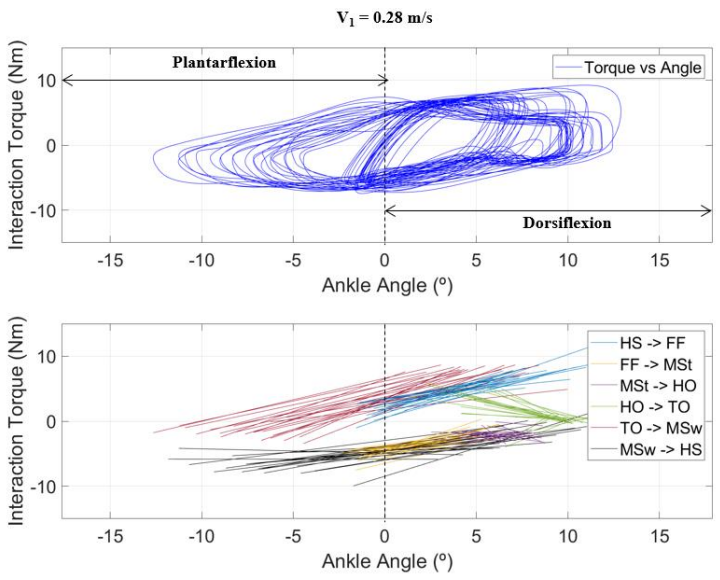

Figure 5: Human-Orthosis interaction torque vs ankle angle (above) and the interaction stiffness estimated by the linear model (below) for one trial of a male subject walking on the treadmill at $0.28 \mathrm{~m} / \mathrm{s}$.

TABLE I. NORMALIZED INTERACTION STIFFNESS VALUES

\begin{tabular}{|c|c|c|c|c|}
\hline \multirow[b]{2}{*}{$\begin{array}{c}\text { Gait } \\
\text { Speed } \\
(\mathbf{m} / \mathbf{s})\end{array}$} & \multirow[b]{2}{*}{$\begin{array}{c}\text { Gait } \\
\text { Phases }\end{array}$} & \multicolumn{3}{|c|}{ Normalized Interaction Stiffness } \\
\hline & & $\begin{array}{c}\text { Interaction } \\
\text { Stiffness }\end{array}$ & $\begin{array}{l}\text { Stance vs } \\
\text { Swing }\end{array}$ & $\begin{array}{c}\text { Single vs } \\
\text { Double } \\
\text { Support }\end{array}$ \\
\hline \multirow{6}{*}{0.280} & $\mathrm{HS} \rightarrow \mathrm{FF}$ & $0.644 \pm 0.383$ & \multirow{4}{*}{$0.514 \pm 0.284$} & $0.644 \pm 0.383$ \\
\hline & $\mathrm{FF} \rightarrow \mathrm{MSt}$ & $0.479 \pm 0.286$ & & \multirow{2}{*}{$0.307 \pm 0.243$} \\
\hline & $\mathrm{MSt} \rightarrow \mathrm{HO}$ & $\begin{array}{l}0.136 \pm \\
0.0523 \\
\end{array}$ & & \\
\hline & $\mathrm{HO} \rightarrow \mathrm{TO}$ & $0.798 \pm 0.172$ & & $0.798 \pm 0.172$ \\
\hline & $\mathrm{TO} \rightarrow \mathrm{MSw}$ & $0.743 \pm 0.198$ & \multirow{2}{*}{$\begin{array}{c}0.782 \pm \\
0.0545\end{array}$} & \multirow{2}{*}{$\begin{array}{l}0.782 \pm \\
0.0545\end{array}$} \\
\hline & $\mathrm{MSw} \rightarrow \mathrm{HS}$ & $0.821 \pm 0.254$ & & \\
\hline \multirow{6}{*}{0.360} & $\mathrm{HS} \rightarrow \mathrm{FF}$ & $0.251 \pm 0.141$ & \multirow{4}{*}{$0.363 \pm 0.329$} & $0.251 \pm 0.141$ \\
\hline & $\mathrm{FF} \rightarrow \mathrm{MSt}$ & $0.295 \pm 0.130$ & & \multirow{2}{*}{$0.183 \pm 0.158$} \\
\hline & $\mathrm{MSt} \rightarrow \mathrm{HO}$ & $\begin{array}{c}0.0707 \pm \\
0.0355 \\
\end{array}$ & & \\
\hline & $\mathrm{HO} \rightarrow \mathrm{TO}$ & $0.836 \pm 0.232$ & & $0.836 \pm 0.232$ \\
\hline & $\mathrm{TO} \rightarrow \mathrm{MSw}$ & $0.584 \pm 0.328$ & \multirow{2}{*}{$\begin{array}{c}0.557 \pm \\
0.0375\end{array}$} & \multirow{2}{*}{$\begin{array}{c}0.557 \pm \\
0.0375\end{array}$} \\
\hline & $\mathrm{MSw} \rightarrow \mathrm{HS}$ & $0.531 \pm 0.275$ & & \\
\hline \multirow{6}{*}{0.440} & $\mathrm{HS} \rightarrow \mathrm{FF}$ & $0.519 \pm 0.352$ & \multirow{4}{*}{$0.566 \pm 0.281$} & $0.519 \pm 0.352$ \\
\hline & $\mathrm{FF} \rightarrow \mathrm{MSt}$ & $0.842 \pm 0.126$ & & \multirow[b]{2}{*}{$0.518 \pm 0.458$} \\
\hline & $\mathrm{MSt} \rightarrow \mathrm{HO}$ & $\begin{array}{c}0.194 \pm \\
0.0525 \\
\end{array}$ & & \\
\hline & $\mathrm{HO} \rightarrow \mathrm{TO}$ & $0.708 \pm 0.412$ & & $0.708 \pm 0.412$ \\
\hline & $\mathrm{TO} \rightarrow \mathrm{MSw}$ & $\begin{array}{c}0.489 \pm \\
0.0552 \\
\end{array}$ & \multirow{2}{*}{$\begin{array}{l}0.468 \pm \\
0.0277\end{array}$} & \multirow{2}{*}{$\begin{array}{l}0.468 \pm \\
0.0277\end{array}$} \\
\hline & $\mathrm{MSw} \rightarrow \mathrm{HS}$ & $0.450 \pm 0.128$ & & \\
\hline
\end{tabular}


According to Table I, we can notice that, for the three gait speeds, the interaction stiffness presented higher normalized values for the final double support phase of the gait cycle, i.e., during $\mathrm{HO} \rightarrow \mathrm{TO}$ event, which indicates that this gait phase is the most critical for subjects. During this phase, the subject applies a force into the orthotic device to propel the foot backwards, beginning the swing phase and the left single support phase. The fact of using the orthosis in unpowered mode introduces an increased effort in the subject. As a quasi-passive device, the orthosis is almost mechanical, presenting inertia to the movement. Moreover, its mass, although not significant, can affect the gait and augment the energy the user is spending. Therefore, it is reasonable that in the left single support phase, when the subject stands all his weight into the left leg, it needs to apply more effort to overcome the inertia that the system is offering. This finding was in accordance with [16] and highlights that the robotic orthosis should be stiffer during this phase, assuming mostly the command, to provide a natural motion to the user. The result is also in accordance with the users' perception since they reported more difficulty in performing the TO event while walking with the unpowered orthosis.

In the second phase of single support and swing phase for the right leg (from TO to a new HS), the normalized value of interaction stiffness decreases as the gait speed increases, which can indicate that, with a higher velocity and, thus, a higher limb acceleration, the user can overcome more easily the inertia and friction that the robotic orthosis is offering in unpowered mode. Therefore, there is no need for higher values of normalized interaction stiffness for performing the correct gait pattern.

\section{B. Assist-as-needed Impedance Control}

In this work, we propose and validate an adaptive impedance control strategy as an AAN strategy for an ankle robotic orthosis in which the robotic device assists the user when needed. Moreover, it encourages the active participation of the user in the therapy session by changing the interaction stiffness with the robotic orthosis. The human-orthosis interaction torque was considered the users' motion intention and effort indicator.

Fig. 6 displays the outcomes for one representative subject walking with the orthotic device at both $0.28 \mathrm{~m} / \mathrm{s}$ and $0.44 \mathrm{~m} / \mathrm{s}$. Analysing Fig. 6, we can notice that, with the adaptive impedance control, the subjects were able to follow the reference trajectory pattern. The similarity to the reference trajectory was achieved since the adaptive impedance control guides the users' motion while adjusts the assistance considering their active participation. Moreover, in some strides, the users were able to overcome the reference trajectory magnitude, highlighting the suitability of this control to enable slight deviations from the reference trajectory considering the users' motion intention. With a lower value of $k$, i.e., low impedance, it is noticeable a lower reference torque (box $\mathbf{B}$ in Fig. 6) for both gait speeds. Even so, the user was able to overcome the reference trajectory due to his/her non-zero interaction with the robotic orthosis.

Fig. 6 also enhances the aptness of the adaptive impedance control as an AAN strategy. During swing and mainly during the TO event, we can see that the reference torque, enhanced by the boxes $\mathbf{B}$, assumes a value different from zero. This indicates that the user needs assistance to correct his/her gait pattern; thus, the robotic orthosis provides the required assistance to tackle this necessity. During the stance phase, the reference torque is almost zero (box $\mathbf{A}$ in Fig. 6) since the error between the reference trajectory and the real angle is lower. As such, the orthosis does not need to assist during this phase.

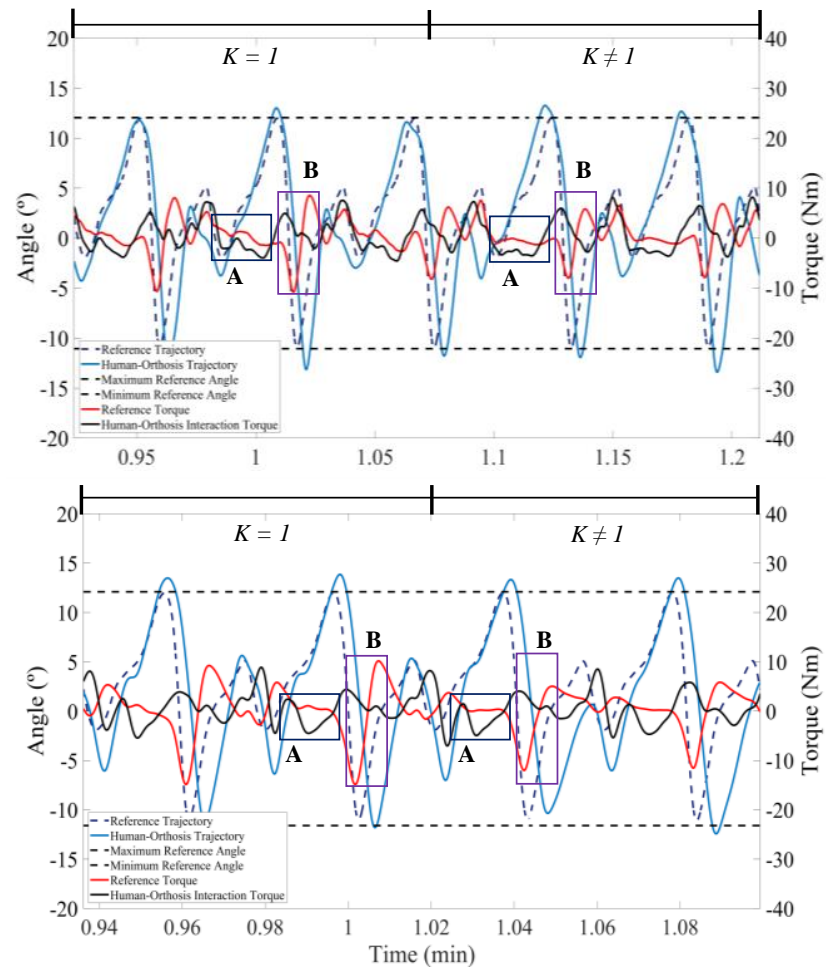

Figure 6: Outcome of one subject walking at $0.28 \mathrm{~m} / \mathrm{s}$ (above) and $0.44 \mathrm{~m} / \mathrm{s}$ (below) with interaction stiffness at $1.0 \mathrm{Nm} /{ }^{\circ} \mathrm{kg}$ and after real-time modifications according to Table I. Black (A) and purple (B) boxes enhance the reference torque generated in stance and swing phases, respectively.

Fig. 7 displays the outcome while the subject was standing his foot on the air, minimizing his interaction with the device. We found that the human-orthosis real angle decreases spontaneously given the lower human-robot interaction. These results reinforce that this strategy is interaction-dependent and enhances its capacity for promoting a user-command assist-asneeded strategy.

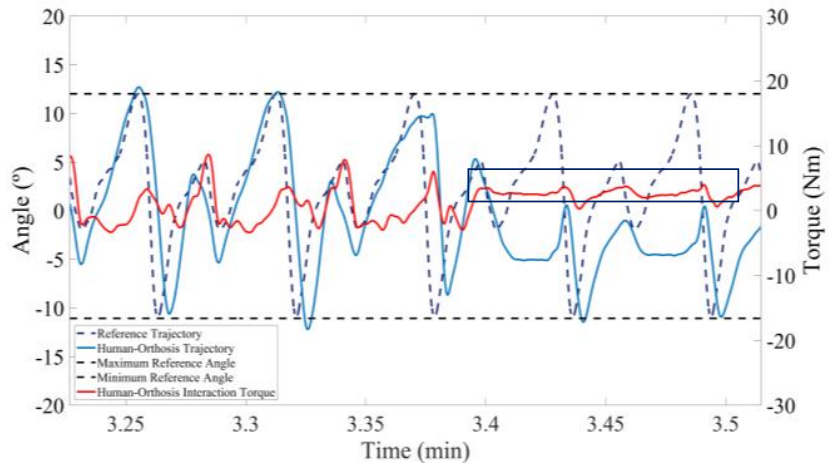

Figure 7: Outcome of one subject walking at $0.28 \mathrm{~m} / \mathrm{s}$ with a reduced interaction with the orthotic device. The box enhances the time in which the user minimized his interaction with the ankle robotic orthosis.

The AAN impedance control strategy presented a delay of $183 \pm 44.1 \mathrm{~ms}$ for $0.28 \mathrm{~m} / \mathrm{s}$ and $225 \pm 25.9 \mathrm{~ms}$ for $0.44 \mathrm{~m} / \mathrm{s}$. This 
value presents some fluctuations between subjects and speed, that is explained by the different interaction that each user produces. For comparison purposes, we implemented a trajectory tracking control strategy, and we found a maximum delay of $250 \mathrm{~ms}$. The result with the AAN impedance control represents an improvement of $27 \%$ and $10 \%$ for $0.28 \mathrm{~m} / \mathrm{s}$ and $0.44 \mathrm{~m} / \mathrm{s}$, respectively, in comparison with the trajectory tracking control. The trajectory tracking control strategy does not consider the human-orthosis interaction torque for the actuator's control. In [16], it was demonstrated that in a trajectory tracking control mode, the gait pattern is not affected by the interaction torque independently of its magnitude. The interaction torque can be seen as the users' performance indicator, evaluating if the user is following or contradicting the imposed trajectory. However, with the proposed control, the interaction torque is a control variable, allowing assistance based on the user's effort and participation.

As the interaction stiffness was modified in real-time, the subjects were able to identify the changes in the orthosis' behaviour. Some of them felt the system "stiffer" after the realtime modifications in the interaction stiffness, in a way that they felt they had to perform more effort to maintain the same walking pattern. On the other hand, other participants reported that they felt the system more compliant in a way that they were able to perform their preferred gait. In fact, decreasing the values of interaction stiffness produced two effects in the users' perception that, at a first view, can be seen as oppositions. However, the two opinions are related. Regarding the orthosis' perspective, reducing the interaction stiffness promotes a more compliant behaviour, not imposing a reference trajectory and giving more freedom. However, in the user's logic, it can be interpreted as a stiffer movement due to the inertia that the system offers as it becomes progressively mechanical. Nevertheless, the users considered this strategy more comfortable in comparison with the trajectory tracking control since they were able to command the orthosis. Moreover, they felt their participation was crucial to provide a more natural motion.

\section{Future Perspectives}

From a future perspective, we will integrate a real-time adaptive trajectory control strategy to provide personalized assistance, modifying the angle trajectory to fulfil the users' necessities. Moreover, the interconnection of the adaptive impedance control and the adaptive trajectory control will be assured to have a fully assistive orthosis, providing assistance sustained in effort, active participation and tailored to each enduser. Future perspectives also include the use of EMG sensors to quantitively assess the muscular strength recovery to better adapt the interaction stiffness. The use of non-linear models and reinforcement learning techniques for real-time stiffness estimation will also be tackled. At last, the clinical validation of the assist-as-needed impedance control will be performed.

\section{CONCLUSION}

This work presents and validates an AAN impedance control strategy for an ankle robotic orthosis. This strategy successfully guides the user's ankle trajectory while adjusting the assistance considering his motion intention (as increases the ankle trajectory) and active participation through human-robot interaction torque. Findings indicate that the real-time adaptation of interaction stiffness yields a fully assistive strategy, capable of gradually passing from a passive state to a more active state; thus, resulting in AAN gait training. In comparison with the trajectory tracking control, we found an improvement in the control's delay of $27 \%$ for $0.28 \mathrm{~m} / \mathrm{s}$ and $10 \%$ for $0.44 \mathrm{~m} / \mathrm{s}$, explained by the active human-orthosis interaction. All subjects reported more freedom to walk with the proposed strategy since they felt they command the robotic orthosis. Therefore, the robotic orthosis is now capable of proving assistance tailored to the end-user needs.

\section{REFERENCES}

[1] W. Meng, Q. Liu, Z. Zhou, Q. Ai, B. Sheng, and S. S. Xie, "Recent development of mechanisms and control strategies for robot-assisted lower limb rehabilitation," Mechatronics, vol. 31, pp. 132-145, 2015.

[2] W. Johnson, O. Onuma, M. Owolabi, and S. Sachdev, "Stroke: A global response is needed," Bull. World Health Organ., vol. 94, no. 9, pp. 634A635A, 2016.

[3] L. N. Awad et al., "A soft robotic exosuit improves walking in patients after stroke," Sci. Transl. Med., vol. 9, no. 400, 2017.

[4] S. Kramer, L. Johnson, J. Bernhardt, and T. Cumming, "Energy Expenditure and Cost during Walking after Stroke: A Systematic Review," Arch. Phys. Med. Rehabil., vol. 97, no. 4, pp. 619-632.e1, 2016.

[5] R. Riener, L. Lünenburger, S. Jezernik, M. Anderschitz, G. Colombo, and V. Dietz, "Patient-cooperative strategies for robot-aided treadmill training: First experimental results," IEEE Trans. Neural Syst. Rehabil. Eng., vol. 13, no. 3, pp. 380-394, 2005.

[6] B. M. Fleerkotte, B. Koopman, J. H. Buurke, E. H. F. Van Asseldonk, H. Van Der Kooij, and J. S. Rietman, "The effect of impedance-controlled robotic gait training on walking ability and quality in individuals with chronic incomplete spinal cord injury: An explorative study," $J$. Neuroeng. Rehabil., vol. 11, no. 1, pp. 1-15, 2014.

[7] S. Hussain, S. Q. Xie, and P. K. Jamwal, "Adaptive impedance control of a robotic orthosis for gait rehabilitation," IEEE Trans. Cybern., vol. 43, no. 3, pp. 1025-1034, 2013.

[8] V. Rajasekaran, J. Aranda, and A. Casals, "Adaptive walking assistance based on human-orthosis interaction," IEEE Int. Conf. Intell. Robot. Syst., pp. 6190-6195, 2015.

[9] M. R. Tucker et al., "Control strategies for active lower extremity prosthetics and orthotics: A review," J. Neuroeng. Rehabil., vol. 12, no. $1,2015$.

[10] D. A. Winter, Biomechanics and Motor Control of Human Movement, 4th Editio., vol. 2nd. Hoboken, New Jersey: John Wiley \& Sons, Inc., 2009.

[11] Neville Hogan, "Impedance Control: An Approach to Manipulation," J. Dyn. Syst. Meas. Control, no. 107, pp. 1-24, 1985.

[12] M. Bortole, "Robotic Exoskeleton With an Assist-as-Needed Control Strategy for Gait Rehabilitation After Stroke," Universidad Carlos III de Madrid, 2014.

[13] K. Shamaei, G. S. Sawicki, and A. M. Dollar, "Estimation of QuasiStiffness and Propulsive Work of the Human Ankle in the Stance Phase of Walking," PLoS One, vol. 8, no. 3, 2013.

[14] K. Molugaram, G. S. Rao, K. Molugaram, and G. S. Rao, "Chapter 5 Curve Fitting," in Statistical Techniques for Transportation Engineering, 2017, pp. 281-292.

[15] J. Figueiredo, P. Félix, L. Costa, J. C. Moreno, and C. P. Santos, "Gait Event Detection in Controlled and Real-Life Situations: Repeated Measures from Healthy Subjects," IEEE Trans. Neural Syst. Rehabil. Eng., vol. 26, no. 10, pp. 1945-1956, 2018.

[16] J. Figueiredo, P. Felix, C. P. Santos, and J. C. Moreno, "Towards humanknee orthosis interaction based on adaptive impedance control through stiffness adjustment," in 2017 International Conference on Rehabilitation Robotics (ICORR), 2017, pp. 406-411. 
